\title{
One nucleon transfer operator and nuclear supersymmetry
}

\author{
J. Barea, ${ }^{1}$ C. E. Alonso, ${ }^{2}$ J. M. Arias, ${ }^{2}$ and J. Jolie ${ }^{3}$ \\ ${ }^{1}$ Instituto de Ciencias Nucleares, Apartado 70-543, UNAM, 04510 México, D.F. México. \\ ${ }^{2}$ Departamento de Física Atómica, Molecular y Nuclear, Facultad de Física, Universidad de Sevilla, Apartado 1065, E-41080 Sevilla, Spain \\ ${ }^{3}$ Institut für Kernphysik, Universitat zu Köln, D-50937 Köln, Germany
}

(Received 4 November 2004; published 27 January 2005)

\begin{abstract}
The appropriate use of the interacting boson-fermion model one nucleon transfer operator in connection with nuclear supersymmetries is discussed. We emphasize that care must be taken in using the same coupling order, either $l-s$ or $s-l$, in the odd particle creation operator appearing in the one nucleon transfer operator and in the wave function of the odd-A nucleus. As an example, we have recalculated consistently the one nucleon transfer strengths for the ${ }^{196} \mathrm{Pt} \rightarrow{ }^{195} \mathrm{Pt}$ one neutron pickup reaction which is the best known example of dynamical nuclear supersymmetry. In addition, we present for the same reaction the results of several calculations considering different truncations in the boson-fermion expansion of the fermion creation operator.
\end{abstract}

DOI: 10.1103/PhysRevC.71.014314

PACS number(s): 21.60.Fw, 21.10.Jx, 25.45.Hi, 23.40.Hc

\section{INTRODUCTION}

The occurrence of supersymmetry in nuclei (n-SUSY) [1-3] has been a matter of interest during the last 20 years [4-6]. During this time the theoretical and experimental effort for identifying some nuclei as examples of the realization of n-SUSY was mainly concentrated in the comparison of the predicted and observed level schemes. Recently, however, new experimental facilities have allowed detailed measurements of nuclear properties in the Pt region [7-10], particularly one nucleon transfer intensities. These experimental data have allowed more stringent testing of the idea of n-SUSY and have provided strong evidence of its existence in nature. Consequently, the interest in the exploration of n-SUSY has been renewed.

There are two kinds of n-SUSY schemes depending on whether the proton-neutron degree of freedom is explicitly considered or not. In the simpler second case, two neighboring even-even and odd-even nuclei are described simultaneously with the same Hamiltonian [1,11]. In the richer, and more interesting, first case a quadruplet of neighboring nuclei, even-even, odd-even, even-odd, and odd-odd, are described with the same Hamiltonian. The corresponding n-SUSY is called extended n-SUSY [3]. The possibility of describing so many experimental data with a reduced number of parameters has encouraged nuclear physicists to look for examples of nuclei that approximate the n-SUSY scheme. In addition, since dynamical n-SUSYs are exactly solvable, the help given in the understanding of nuclear structure is invaluable. For this reason, there is continuous feedback between theory and experiment. Incidentally, the dynamical n-SUSY description can also be used as a starting point for getting better fits to experimental data in perturbed calculations [12].

As mentioned above, there is presently strong evidence of the realization of n-SUSY in the Pt-Au nuclei. In this region the fermion space can be truncated to the $3 p_{1 / 2}, 3 p_{3 / 2}$, and $2 f_{5 / 2}$ orbitals for the neutrons and to the $2 d_{3 / 2}$ orbital for the protons. Consequently, the appropriate extended dynamical n-SUSY is $U_{v}(6 / 12) \otimes U_{\pi}(6 / 4)$. In it one considers the coupling of a neutron, which can occupy $j_{v}=1 / 2,3 / 2$, and $5 / 2$ orbits, and a $j_{\pi}=3 / 2$ proton to an $O(6)$ core. In this paper we concentrate on the evaluation of one neutron transfer strengths within $\mathrm{n}$-SUSY and the interacting boson-fermion approximation (IBFA) model [11] since they give valuable information about the wave functions of the states involved. In Sec. II we address the importance of being consistent in the coupling order adopted, both in the operators and in the wave functions, for the orbital and spin angular momenta of the odd particle. The use of different coupling orders in operators and wave functions gives rise to relative phases that make the calculation incorrect. We illustrate this point with a simple example and present the correct results for the spectroscopic strengths for the ${ }^{196} \mathrm{Pt} \rightarrow$ ${ }^{195} \mathrm{Pt}$ one neutron pickup reaction. In Sec. III we present the evaluation of one nucleon transfer spectroscopic strengths for the same reaction using three different truncations of the boson-fermion image of the one nucleon transfer operator (the associated fermionic shell-model operator). We consider first the $a_{j}^{\dagger}$ operator. The second operator used is the one usually proposed in IBFA that includes the next term in the boson-fermion expansion. Finally, the third one includes one additional term in the expansion, as proposed recently [13]. The calculations performed with this last operator are the first actual application of the formalism presented in Ref. [13]. Comparison of these results with experimental data shows the importance of including higher order terms in the boson-fermion image of the one nucleon transfer operator to improve the agreement with the data. Finally, Sec. IV presents the conclusions of this work.

\section{CONSISTENT COUPLING ORDER IN OPERATORS AND STATES}

In this section we want to draw attention to the care that must be taken to avoid inconsistencies in the coupling order of angular momenta in general. In particular, we are going to focus on the case of the evaluation of spectroscopic strengths when using an n-SUSY scheme.

To obtain the n-SUSY wave functions, one has to specify from the beginning the coupling order of orbital $l$ and spin $s$ 
angular momenta in the fermion creation operator. The choice of this order is irrelevant as long as one keeps it consistent throughout the calculation. In the calculations presented in $[9,10]$ the wave functions were obtained from n-SUSY while the transfer operator was taken from the IBFA. For this reason, one should make sure that the $a_{j}^{\dagger}$ chosen have been obtained using the same coupling criteria as in the n-SUSY wave functions. This consistency is difficult to know just by looking at the expressions of wave functions and operators since the only label commonly used is the total angular momentum $j$. No track is usually conserved on the $l-s$ or $s-l$ intermediate coupling.

Let us illustrate our point by discussing the use of the semimicroscopically derived IBFA one nucleon transfer operator $[14,15]$ in connection with the n-SUSY wave functions. The IBFA image of the shell-model one fermion creation operator $c_{j}^{\dagger}$ is in general an infinite expansion in terms of the boson operators $s^{\dagger}, d^{\dagger}$ and an "ideal" fermion operator $a_{j}^{\dagger}$ acting in the boson-fermion space. A truncation of this expansion $T_{j}^{\dagger}$ was proposed in Ref. [14] using the generalized seniority scheme and considering terms including up to one $d$-boson which change generalized seniority $\widetilde{v}$ in one unit. Under these conditions, the IBFA image of the one fermion creation operator for transfer reactions conserving the boson number is obtained [14,15] within a scheme called IBFA-2 [11] in which the neutron-proton degree of freedom is explicitly taken into account (the simpler version of the IBFA model which does not include explicitly the neutron-proton degree of freedom is known as IBFA-1) as

$$
\begin{aligned}
\left(T_{j}^{\dagger}\right)_{\rho}^{(\mathrm{IBFA}-2)}= & \left\{u_{j} a_{j}^{\dagger}-\sum_{j^{\prime}} \sqrt{\frac{10}{(2 j+1) N}} v_{j}\right. \\
& \left.\times \frac{Q_{j^{\prime} j}\left(u_{j} v_{j^{\prime}}+v_{j} u_{j^{\prime}}\right)}{N_{\beta}} s^{\dagger}\left(\widetilde{d} a_{j^{\prime}}^{\dagger}\right)^{(j)}\right\}_{\rho}
\end{aligned}
$$

where $\rho$ stands for neutron or proton (depending on the nature of the transferred particle), the $u_{j}$ are related to the occupation probabilities, $N_{\rho}$ is the total number of $\rho$ bosons, $Q_{j^{\prime} j}$ are the matrix elements of the quadrupole operator between states $j^{\prime}$, $j$ and $N_{\beta}=\left(\sum_{j j^{\prime}} \beta_{j^{\prime} j}^{2}\right)^{\frac{1}{2}}$ with

$$
\beta_{j^{\prime} j}=Q_{j^{\prime} j}\left(u_{j} v_{j^{\prime}}+v_{j} u_{j^{\prime}}\right) .
$$

Usually the odd-nucleon creation operator $a_{j}^{\dagger}$ has only one label $j$, with the corresponding $l$ and $s$ hidden in some way. The coupling scheme is unimportant when the explicit expression for $Q_{j^{\prime} j}$ is not stated. However, when the fermion quadrupole operator $q^{(2)}$ is written in terms of the actual shellmodel one fermion creation and annihilation operators $c_{j}^{\dagger}$ and $\widetilde{c}_{j}$ as

$$
q^{(2)}=\sum_{j j^{\prime}} Q_{j^{\prime} j}\left(c_{j^{\prime}}^{\dagger} \widetilde{c}_{j}\right)^{(2)}=\sum_{j j^{\prime}}\left\langle l^{\prime} \frac{1}{2} j^{\prime}\left\|Y^{(2)}\right\| l \frac{1}{2} j\right\rangle\left(c_{j^{\prime}}^{\dagger} \widetilde{c}_{j}\right)^{(2)}
$$

and $Q_{j^{\prime} j}=\left\langle l^{\prime} \frac{1}{2} j^{\prime}\left\|Y^{(2)}\right\| l \frac{1}{2} j\right\rangle$, then, $l-s$ coupling is adopted for $c_{j}^{\dagger}$ and $\widetilde{c}_{j}$ and, consequently, for the $a_{j}^{\dagger}$ in the IBFA transfer operator. One could choose alternatively the $s-l$ coupling just by taking $Q_{j^{\prime} j}=\left\langle\frac{1}{2} l^{\prime} j^{\prime}\left\|Y^{(2)}\right\| \frac{1}{2} l j\right\rangle$. The difference between the two elections of $Q_{j^{\prime} j}$ is a phase factor $(-1)^{j-j^{\prime}}$ which is +1 when one considers just a single $j$ case but introduces relative phases if there are several open orbits as in the case of the $U_{v}(6 / 12) \otimes U_{\pi}(6 / 4)$ n-SUSY.

On the other hand, when one expresses the n-SUSY wave functions in the coupled notation for $U_{v}(6 / 12) \otimes U_{\pi}(6 / 4)$ one has to select at the beginning $\tilde{l}-\tilde{s}$ or $\tilde{s}-\tilde{l}$ coupling in the $a_{j}^{\dagger}$ operator to define the generators of the different algebras involved [16], where $\tilde{l}$ and $\tilde{s}$ indicate pseudo-orbital and pseudospin angular momenta, respectively. In the coupled notation, the different components of the wave functions are labeled, in addition to the boson labels, by the odd fermion label $j$ that in fact includes the coupling order. In Ref. [16], $\tilde{l}-\tilde{s}$ coupling was selected and $l-s$ coupling is implied automatically.

To illustrate our point, we refer to the calculation shown in the appendix of Ref. [9]. The operator chosen in Ref. [9] to analytically calculate the transfer matrix element $T_{f i}\left(0_{1}^{+} \rightarrow\right.$ $1 / 2-$ ) between ${ }^{196} \mathrm{Pt}$ and ${ }^{195} \mathrm{Pt}$ is given in Eq. (4) of that paper, apart from a global factor, as

$$
\begin{aligned}
\left(T_{j}^{\dagger}\right)^{(\mathrm{IBFA})} & =v_{j} a_{j}^{\dagger}-\sum_{j^{\prime}} \sqrt{\frac{10 N_{v}}{(2 j+1) N^{2}}} u_{j} \\
& \times \frac{\left\langle\frac{1}{2} l^{\prime} j^{\prime}\left\|Y^{(2)}\right\| \frac{1}{2} l j\right\rangle\left(u_{j} v_{j^{\prime}}+v_{j} u_{j^{\prime}}\right)}{N_{\beta}} s^{\dagger}\left(\widetilde{d} a_{j^{\prime}}^{\dagger}\right)^{(j)} .
\end{aligned}
$$

This operator is obtained from Eq. (1) when the hole nature of the odd neutron and the projection from IBFA-2 to IBFA-1 (through a multiplying factor $N_{v} / N$ in the second term of the right-hand side) are taken into account and using $Q_{j^{\prime} j}=$ $\left\langle\frac{1}{2} l^{\prime} j^{\prime}|| Y^{(2)} \| \frac{1}{2} l j\right\rangle$. Consequently, the "hidden" coupling is $s-l$, i.e., $a_{j}^{\dagger}=a_{\left(\frac{1}{2} l\right) j}^{\dagger}$ and $a_{j^{\prime}}^{\dagger}=a_{\left(\frac{1}{2} l^{\prime}\right) j^{\prime}}^{\dagger}$.

The $1 / 2_{2}^{-}$state reads (Table XV in Ref. [16])

$$
\begin{aligned}
\left|\frac{1^{-}}{2}\right\rangle= & \sqrt{\frac{3}{5}}\left[|[N]\langle N\rangle(1) 2\rangle \times a_{3 / 2}^{\dagger}\right]^{\left(\frac{1}{2}\right)} \\
& +\sqrt{\frac{2}{5}}\left[|[N]\langle N\rangle(1) 2\rangle \times a_{5 / 2}^{\dagger}\right]^{\left(\frac{1}{2}\right)},
\end{aligned}
$$

where the $a_{j}^{\dagger}$ are $a_{\left(l \frac{1}{2}\right) j}^{\dagger}$ as stated in Ref. [16]. Then we see that before calculating matrix elements of the transfer operator (3) between states in the even-even nucleus and this n-SUSY state (4) in the odd-even nucleus we have to change the coupling order either in the operator or in the state. For the change in the transfer operator (3) it is enough to include in the second term of the right-hand side of Eq. (3) a phase $(-1)^{j^{\prime}-j}$ which makes the operator suitable to be used with the wave functions obtained in Ref. [16]. To perform a consistent calculation of $T_{f i}\left(0_{1}^{+} \rightarrow 1 / 2_{2}^{-}\right)$the quantity to be evaluated according to Eq. (A3) in Ref. [9] is

$$
\frac{\beta_{\frac{3}{2} \frac{1}{2}}}{2}+\frac{\beta_{\frac{5}{2} \frac{1}{2}}}{\sqrt{6}} .
$$

If we use the occupation probabilities provided by the n-SUSY [17] $\left(v_{1 / 2}^{2}=0.1, v_{3 / 2}^{2}=v_{5 / 2}^{2}=0.6\right), \beta_{\frac{3}{2} \frac{1}{2}}=-\sqrt{\frac{2}{3}} \beta_{\frac{5}{2} \frac{1}{2}}$ and 
TABLE I. Summary of experimental results [9] for ${ }^{195} \mathrm{Pt}$ from transfer experiments compared to the results of our theoretical calculation.

\begin{tabular}{|c|c|c|c|c|c|c|}
\hline \multicolumn{3}{|c|}{ Expt. } & \multicolumn{4}{|c|}{ Theory } \\
\hline Energy (keV) & $J^{\pi}$ & $G_{l j}\left(10^{-2}\right)$ & Energy (keV) & $J^{\pi}$ & $G_{l j}^{\mathrm{a}}\left(10^{-2}\right)$ & $G_{l j}^{\mathrm{b}}\left(10^{-2}\right)$ \\
\hline 0.0 & $1 / 2^{-}$ & $78.12(22)$ & 0.0 & $1 / 2^{-}$ & 69.72 & 95.26 \\
\hline $99.5(6)$ & $3 / 2^{-}$ & $88.72(28)$ & 162.0 & $3 / 2^{-}$ & 133.01 & 109.33 \\
\hline $129.5(6)$ & $5 / 2^{-}$ & $209.2(6)$ & 179.0 & $5 / 2^{-}$ & 226.97 & 186.58 \\
\hline $199.2(6)$ & $3 / 2^{-}$ & $12.76(12)$ & 244.2 & $3 / 2^{-}$ & 0.00 & 0.00 \\
\hline $212.4(6)$ & $3 / 2^{-}$ & $20.60(12)$ & 253.0 & $3 / 2^{-}$ & 34.25 & 53.63 \\
\hline $223.5(7)$ & $1 / 2^{-}$ & $14.6(8)$ & 234.0 & $1 / 2^{-}$ & 0.00 & 0.00 \\
\hline $238.7(6)$ & $5 / 2^{-}$ & $34.98(24)$ & 270.0 & $5 / 2^{-}$ & 58.56 & 91.55 \\
\hline $259.4(6)$ & $13 / 2^{+}$ & $356(25)$ & & & & \\
\hline $389.5(7)$ & $5 / 2^{-}$ & $1.44(6)$ & 317.2 & $5 / 2^{-}$ & 0.00 & 0.00 \\
\hline $419.5(7)$ & $3 / 2^{-}$ & $0.76(4)$ & 475.8 & $3 / 2^{-}$ & 0.00 & 4.98 \\
\hline $432.7(6)$ & $9 / 2^{+}$ & $44.8(4)$ & & & & \\
\hline $450.0(7)$ & $7 / 2^{-}$ & $1.52(16)$ & 341.0 & $7 / 2^{-}$ & & \\
\hline $455.6(7)$ & $5 / 2^{-}$ & $1.50(12)$ & 492.8 & $5 / 2^{-}$ & 0.00 & 8.47 \\
\hline $507.9(6)$ & $7 / 2^{-}$ & $51.12(24)$ & 595.0 & $7 / 2^{-}$ & & \\
\hline $524.6(6)$ & $3 / 2^{-}$ & $0.76(4)$ & 566.8 & $3 / 2^{-}$ & 0.00 & 2.66 \\
\hline $543.9(6)$ & $5 / 2^{-}$ & $2.76(6)$ & 583.8 & $5 / 2^{-}$ & 0.00 & 4.54 \\
\hline $562.6(7)$ & $9 / 2^{-}$ & $31.4(7)$ & 625.6 & $9 / 2^{-}$ & & \\
\hline $590.896(5)^{\mathrm{c}}$ & $3 / 2^{-}$ & & 558.0 & $3 / 2^{-}$ & 0.00 & 0.00 \\
\hline $612.0(6)$ & $7 / 2^{-}$ & $51.36(24)$ & 686.0 & $7 / 2^{-}$ & & \\
\hline $628.8(7)$ & $1 / 2^{-}$ & $0.60(4)$ & 547.8 & $1 / 2^{-}$ & 0.00 & 0.00 \\
\hline $632.1(5)^{\mathrm{c}}$ & $1 / 2^{-}, 3 / 2^{-}$ & & 580.4 & $3 / 2^{-}$ & 0.00 & 0.00 \\
\hline $664.2(6)$ & $5 / 2^{-}$ & $10.92(12)$ & 597.4 & $5 / 2^{-}$ & 0.00 & 0.00 \\
\hline $667.1(5)^{\mathrm{c}}$ & $\left(9 / 2^{-}\right)$ & & 716.6 & $9 / 2^{-}$ & & \\
\hline $678.4(8)$ & $5 / 2^{-}$ & $0.79(6)$ & 631.0 & $5 / 2^{-}$ & 0.00 & 0.00 \\
\hline $695.3(6)$ & $7 / 2^{-}$ & $10.64(16)$ & 654.8 & $7 / 2^{-}$ & & \\
\hline $739.5(6)$ & $1 / 2^{-}$ & $11.04(6)$ & 668.6 & $1 / 2^{-}$ & 34.11 & 12.72 \\
\hline $766.7(6)$ & $7 / 2^{-}$ & $14.80(16)$ & 699.6 & $7 / 2^{-}$ & & \\
\hline $794.5(6)$ & $13 / 2^{+}$ & $130.9(11)$ & & & & \\
\hline $793.0(10)^{\mathrm{c}}$ & $3 / 2^{-}$ & & 850.4 & $1 / 2^{-}$ & 0.00 & 0.00 \\
\hline $814.9(6)$ & $9 / 2^{-}$ & $119.3(14)$ & 730.2 & $9 / 2^{-}$ & & \\
\hline $821.9(12)$ & $5 / 2^{+}$ & $0.96(6)$ & & & & \\
\hline $873.8(6)$ & $7 / 2^{-}$ & $14.21(16)$ & 968.6 & $7 / 2^{-}$ & & \\
\hline $895.0(9)$ & $9 / 2^{-}$ & $6.10(40)$ & 786.2 & $9 / 2^{-}$ & & \\
\hline $916.0(6)$ & $7 / 2^{-}$ & $24.16(16)$ & 1013.4 & $7 / 2^{-}$ & & \\
\hline $927.9(6)$ & $3 / 2^{-}$ & 7.92(8) & 921.6 & $3 / 2^{-}$ & 5.94 & 1.93 \\
\hline $930.71^{\mathrm{c}}$ & $9 / 2^{-}$ & & 1044.0 & $9 / 2^{-}$ & & \\
\hline $970.6(6)$ & $7 / 2^{-}$ & $29.76(16)$ & 1059.6 & $7 / 2^{-}$ & & \\
\hline $1010.4(7)$ & $5 / 2^{-}$ & $5.34(12)$ & 938.6 & $5 / 2^{-}$ & 10.14 & 3.33 \\
\hline 1047.1(7) & & $40.80(24)$ & 1104.4 & $7 / 2^{-}$ & & \\
\hline $1068.8(7)$ & $9 / 2^{-}$ & $20.4(8)$ & 1135.0 & $9 / 2^{-}$ & & \\
\hline $1079.7(7)$ & $5 / 2^{-}$ & $6.18(12)$ & 1015.8 & $5 / 2^{-}$ & 0.00 & 0.00 \\
\hline $1095.5(7)$ & $3 / 2^{-}$ & $34.44(12)$ & 976.4 & $3 / 2^{-}$ & 0.00 & 0.00 \\
\hline 1111.2(7) & $7 / 2^{-}$ & $2.72(16)$ & 1073.2 & $7 / 2^{-}$ & & \\
\hline 1132.3(7) & $1 / 2^{-}$ & $3.30(4)$ & 966.2 & $1 / 2^{-}$ & 0.00 & 0.00 \\
\hline $1150.0(8)$ & $9 / 2^{+}$ & $1.2(6)$ & & & & \\
\hline 1155.7(8) & $5 / 2^{-}$ & $8.58(24)$ & 1049.4 & $5 / 2^{-}$ & 0.00 & 0.00 \\
\hline $1160.38^{c}$ & $1 / 2^{-}, 3 / 2^{-}$ & & 998.8 & $3 / 2^{-}$ & 0.00 & 0.00 \\
\hline $1175.5(8)$ & $7 / 2^{-}$ & $9.28(16)$ & 1118.0 & $7 / 2^{-}$ & & \\
\hline 1271.2(9) & $3 / 2^{-}$ & $3.40(4)$ & 1235.4 & $3 / 2^{-}$ & 0.00 & 0.60 \\
\hline 1288.3(9) & $1 / 2^{-}$ & $2.56(8)$ & 1350.4 & $1 / 2^{-}$ & 7.68 & 0.00 \\
\hline 1288.3(9) & $5 / 2^{-}$ & $8.22(30)$ & 1252.4 & $5 / 2^{-}$ & 0.00 & 1.06 \\
\hline 1314.1(10) & $5 / 2^{-}$ & $5.76(12)$ & 1360.6 & $5 / 2^{-}$ & 0.00 & 0.15 \\
\hline $1321.0(10)$ & $3 / 2^{-}$ & $0.64(4)$ & 1343.6 & $3 / 2^{-}$ & 0.00 & 0.07 \\
\hline 1328.1(10) & $13 / 2^{+}$ & $33.7(10)$ & & & & \\
\hline
\end{tabular}


TABLE I. (Continued.)

\begin{tabular}{|c|c|c|c|c|c|c|}
\hline \multicolumn{3}{|c|}{ Expt. } & \multicolumn{4}{|c|}{ Theory } \\
\hline Energy (keV) & $J^{\pi}$ & $G_{l j}\left(10^{-2}\right)$ & Energy (keV) & $J^{\pi}$ & $G_{l j}^{\mathrm{a}}\left(10^{-2}\right)$ & $G_{l j}^{\mathrm{b}}\left(10^{-2}\right)$ \\
\hline $1334.7(4)^{\mathrm{c}}$ & $1 / 2^{-}, 3 / 2^{-}$ & & 1417.2 & $3 / 2^{-}$ & 0.00 & 0.00 \\
\hline $1342.4(13)$ & $5 / 2^{-}, 7 / 2^{-}$ & $0.96(8)$ & 1434.2 & $5 / 2^{-}$ & 0.00 & 0.00 \\
\hline $1348.2(11)$ & $5 / 2^{+}$ & $1.14(6)$ & & & & \\
\hline $1371.9(12)$ & $3 / 2^{-}$ & $1.48(4)$ & 1425.8 & $3 / 2^{-}$ & 0.00 & 0.00 \\
\hline $1380.3(12)$ & $13 / 2^{+}$ & $57.1(11)$ & & & & \\
\hline $1405.0(13)$ & $13 / 2^{+}$ & $50.7(10)$ & & & & \\
\hline $1426.6(14)$ & $7 / 2^{-}$ & $1.52(8)$ & 1354.6 & $7 / 2^{-}$ & & \\
\hline 1437.7(14) & $1 / 2^{-}$ & $1.28(4)$ & 1415.6 & $1 / 2^{-}$ & 0.00 & 0.00 \\
\hline $1445.9(14)$ & $3 / 2^{-}$ & $1.72(4)$ & 1499.4 & $3 / 2^{-}$ & 0.00 & 0.00 \\
\hline $1455.9(14)$ & $7 / 2^{-}$ & $0.56(8)$ & & & & \\
\hline $1464.7(15)$ & $5 / 2^{-}$ & $1.86(12)$ & 1498.8 & $5 / 2^{-}$ & 0.00 & 0.00 \\
\hline $1473.2(15)$ & $3 / 2^{-}$ & $0.48(4)$ & & & & \\
\hline
\end{tabular}

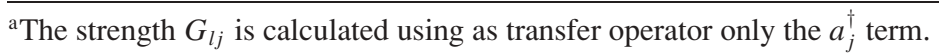

${ }^{\text {b }}$ The strength $G_{l j}$ is calculated using the transfer operator given in Eq. (3) with the inclusion of a phase $(-1)^{j^{\prime}-j}$ in the second term of the right-hand side in order to have the same coupling order in the operator and in the wave functions.

${ }^{c}$ Data from [18] because they were not resolved in [9].

according to (A3) of [9] $T_{f i}\left(0_{1}^{+} \rightarrow \frac{1}{2}^{-}\right)=0$ and not the value calculated in [9]. This value is obtained when, incorrectly, the $\beta_{j^{\prime} j}$ are calculated from the $Q_{j^{\prime} j}$ associated to $s-l$ coupling. It is worth noting that in Ref. [9] the occupation probabilities are obtained from a least square fit to the experimental data and are not those provided by n-SUSY. Nevertheless the above relation for the $\eta$ 's $\beta$ is approximately satisfied and the value obtained for $T_{f i}\left(0_{1}^{+} \rightarrow 1 / 2_{2}^{-}\right)$is of the order of $10^{-4}$ (and the corresponding spectroscopic strength is of the order of $10^{-8}$ ).

In Table I we present the results for the spectroscopic strengths obtained in a consistent way using $l$-s coupling along with the experimental data of Ref. [9] for the ${ }^{196} \mathrm{Pt} \rightarrow{ }^{195} \mathrm{Pt}$ reaction. The calculations are performed using the wave functions provided by the n-SUSY for ${ }^{196} \mathrm{Pt}$ and ${ }^{195} \mathrm{Pt}$ as in Refs. [9,10] and the occupation probabilities provided by n-SUSY as mentioned above. The SUSY labels assigned to each state are the same as in Ref. [9]. In Table I we include two calculations for the spectroscopic strength $G_{l j}$, the first one (in the sixth column) was obtained using as transfer operator only the $a_{j}^{\dagger}$ term. It can be observed that already this simple operator gives the main characteristics of the strengths. The second calculation (in the seventh column) was obtained using the complete operator of Eq. (3) but with the mentioned phase in order to have the same coupling in the operator and in the state. In both cases, the theoretical spectroscopic strengths have been normalized for each $j$ to the experimental sum rules.

All known states up to around $1500 \mathrm{keV}$ are included in the table. The differences observed between the results shown in the last column in Table I and the ones of Ref. [10] are due to inconsistencies in the code IBFFMTR [19] between the coupling order used in the transfer operator and the one used in the wave functions. Changes due to the different election of the occupation probabilities are tiny. The biggest changes occur for the $3 / 2^{-}$states, especially for the third one at $253 \mathrm{keV}$; but one also notices a sizable reduction of fragmentation compared to Ref. [10].

\section{III. ${ }^{196} \mathrm{Pt} \rightarrow{ }^{195} \mathrm{Pt}$ DESCRIBED WITH n-SUSY}

In this section we keep our interest in studying one nucleon transfer spectroscopic strengths by using n-SUSY wave functions and the IBFA transfer operator. Our aim is to look into the differences of the one nucleon transfer strengths induced by different truncations of the transfer operator. We start with the simplest one $\left(a_{j}^{\dagger}\right)$ and then add higher order terms (only the ones that change generalized seniority in one unit) in the boson-fermion expansion.

Again, we consider the reaction ${ }^{196} \mathrm{Pt} \rightarrow{ }^{195} \mathrm{Pt}$. To calculate spectroscopic strengths for this one neutron transfer reaction, we described the nuclei involved with the usual n-SUSY Hamiltonian [3] as

$$
\begin{aligned}
H= & A C_{2}\left[U_{v \pi}^{\mathrm{BF}}(6)\right]+B C_{2}\left[O_{v \pi}^{\mathrm{BF}}(6)\right]+C C_{2}\left[O_{v \pi}^{\mathrm{BF}}(5)\right] \\
& +D C_{2}\left[O_{v \pi}^{\mathrm{BF}}(3)\right]+E C_{2}[\operatorname{Spin}(3)],
\end{aligned}
$$

where $C_{2}[G]$ is the quadratic Casimir operator of the group $G$ belonging to the group chain $U_{v}(6 / 12) \otimes U_{\pi}(6 / 4)$ chosen to describe these nuclei. The parameters appearing in Eq. (5) have been taken from [9] for both nuclei. The choice of the parameters in the Hamiltonian is not important for the calculation of spectroscopic strengths since the eigenstates are given by the quantum numbers labeling the irreducible representations (irreps) of the chain of groups chosen, and they are the same for any choice of parameters. For this reason, any set of parameters given in the literature [7,9] for the description of these nuclei will be good for our calculations. Although the energies will be different for each set, the structure of the wave functions is given by the n-SUSY scheme.

Once the states are fixed with the choice of the Hamiltonian, one has to choose the transfer operator. This operator, for boson 


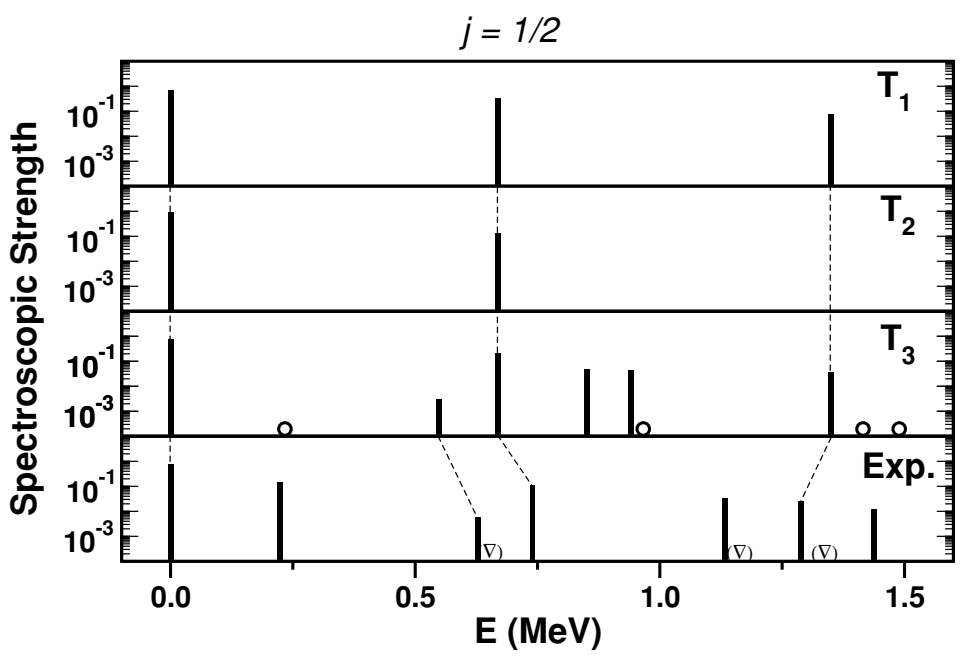

FIG. 1. Spectroscopic strengths for the reaction ${ }^{196} \mathrm{Pt} \rightarrow{ }^{195} \mathrm{Pt}$ for $j=1 / 2$ transfers. The experimental data have been taken from Ref. [9]. The different panels show calculations truncating the boson-fermion expansion of the fermion creation operator at different orders. The labels $T_{1}, T_{2}$, and $T_{3}$ indicate the number of terms retained in the transfer operator (6) as explained in the text. States with spectroscopic strengths smaller that $10^{-4}$ are included in the bottom line of $T_{3}$ and Exp. panels as open circles so as to present the complete experimental and calculated spectroscopy. Experimental states at 632, 1160, and $1334 \mathrm{keV}$ observed in Ref. [18] have the uncertain assignment $1 / 2^{-}, 3 / 2^{-}$; they have been included in both Figs. 1 and 2 as open triangles $(\nabla)$. number conserving reactions, is an expansion of the type [14]

$$
\begin{aligned}
c_{j}^{\dagger}= & A_{j} a_{j}^{\dagger}+\gamma \sum_{j^{\prime}} B_{j j^{\prime}} s^{\dagger}\left(\tilde{d} a_{j^{\prime}}^{\dagger}\right)^{(j)} \\
& +\delta \sum_{L j^{\prime}} C_{L j j^{\prime}}\left[\left(d^{\dagger} \widetilde{d}\right)^{(L)} a_{j^{\prime}}^{\dagger}\right]^{(j)}+\ldots
\end{aligned}
$$

The coefficients $A_{j}$ and $B_{j j^{\prime}}$ were determined in Ref. [14]. The determination of the expansion coefficients $C_{L j j^{\prime}}$ can be done in principle by an extension of the method presented in $[14,15]$. This calculation is cumbersome since fermion states with generalized seniority $\widetilde{v}=3$ are involved and the mapping from the fermion space to the IBFA space is not trivial. Nevertheless, this calculation has been presented recently $[12,13]$ and the values of $C_{L j j^{\prime}}$ can be deduced from the $\phi_{j j^{\prime}}^{J}$ of Ref. [13]. In [12,13] it was shown that the addition of the $\sum_{L j^{\prime}} C_{L j j^{\prime}}\left[\left(d^{\dagger} \widetilde{d}\right)^{(L)} a_{j^{\prime}}^{\dagger}\right]^{(j)}$ terms gives rise to sizable changes in the spectroscopic strengths in the three limits studied. For this reason, it is important to use the extended operator, especially in a reaction used to test the occurrence of n-SUSY.

Up to now, most calculations in the n-SUSY scheme have been performed taking the simplest transfer operator, which includes only the first term in the series (6). We present here calculations with three different truncations of the operator (6). The first one keeps just the first term. The second includes the first and the second terms. Finally, the third calculation is performed with all the terms given explicitly in (6). By comparing the results of different truncations, we will be able to see the relative importance of the various terms and the relevance of the choice of the transfer operator in describing nuclei in the very restricted dynamical n-SUSY scheme. Parameters $\gamma$ and $\delta$ are included in (6) in order to weigh the relative importance of the different terms. We have fitted them to get the best agreement with the experimental data.

The results of the three different calculations for the spectroscopic strengths, along with the experimental data, are presented in Figs. $1-3$ (for $j=1 / 2,3 / 2$, and $5 / 2$, respectively). The logarithmic scales used in the figures are needed because the spectroscopic strengths vary over orders of magnitude. In each figure, $T_{1}, T_{2}$, and $T_{3}$ indicate that the transfer operator used includes the first term of the expansion, the first two terms, and the first three terms, respectively. In the calculation including the first two terms in the transfer operator, the parameter $\gamma$ is taken as 1 . In the

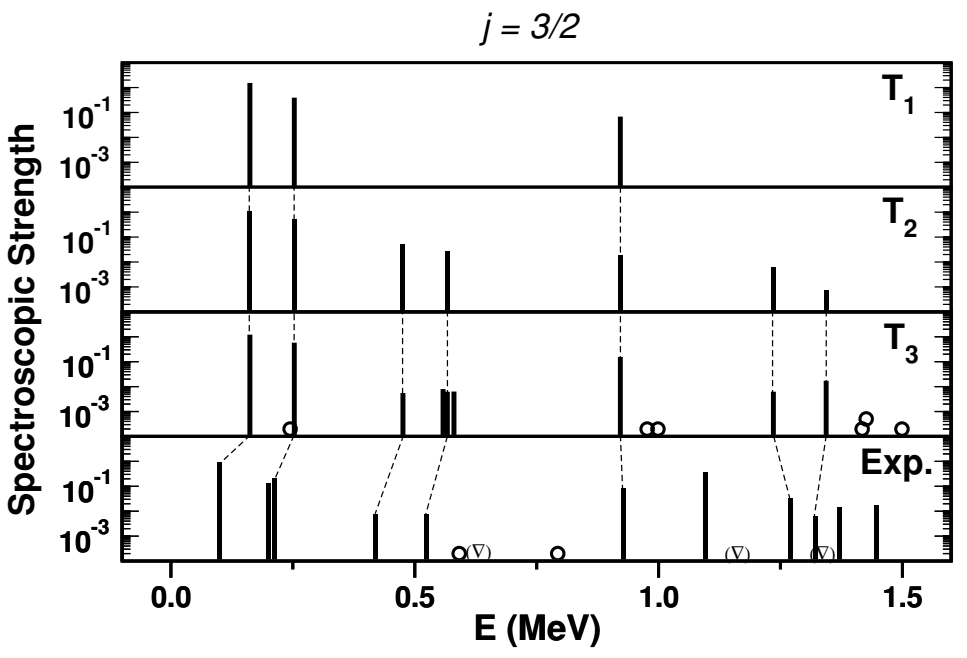

FIG. 2. Same as Fig. 1, but for $j=3 / 2$ transfers. 


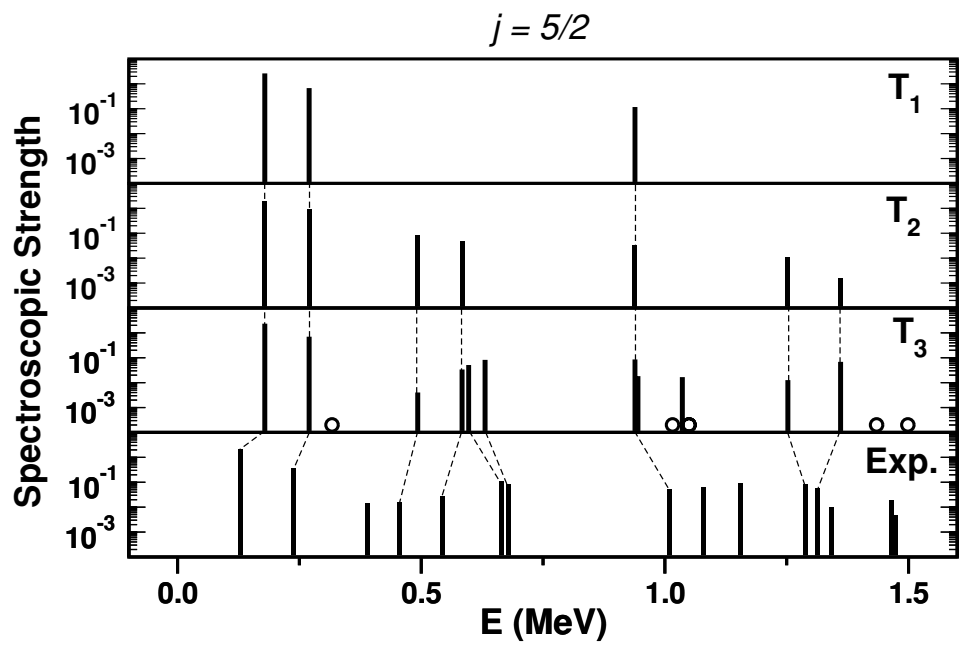

FIG. 3. Same as Fig. 1 , but for $j=5 / 2$ transfers.

calculation including the three terms in the transfer operator, the parameters $\gamma$ and $\delta$ have been fitted using a $\chi^{2}$ fit. The values obtained are $\gamma=0.1157$ and $\delta=2.1454$, showing that in order to describe the experimental data the relative weight of the third term is important.

In Figs. 1-3, we can see that the simplest operator already gives the main observed features but is unable to reproduce the fragmentation observed in the experimental data. The selection rules associated to this operator are very tight. The addition of higher order terms in the expansion (6) improves the description of the spectroscopic strengths. The best results are obtained when the three terms are included. The fragmentation observed experimentally is well reproduced for the three possible $j$ transferred. Still, a detailed observation of Table I suggests that the description could be improved if some changes were made in the assignation of the quantum numbers. Unfortunately, for these states there is not enough information on electromagnetic properties to support these changes. This information would be highly desirable.

The calculations shown in this section have been performed using the IBFA-2 codes ODDPAR, ODDSPEC [20], and ODDSPEC $+[21]$. They could have been performed with IBFA-1 codes as well, since the bosonic part of the wave functions involved is fully symmetric. In that case a projected transfer operator would have been used, as in Ref. [9].

\section{CONCLUSIONS}

This paper has shown the importance of keeping the coupling orders of the orbital and spin angular momenta consistent in all stages of a calculation. In our case, we focused on the calculation of one nucleon transfer reactions in the context of the extended n-SUSY. A careful check of the consistent coupling order of the "hidden" $l$ and $s$ angular momenta in wave functions and operators should be done at all stages of the calculation. If not, phases will appear that will make results incorrect and they will not even fulfill selection rules.

The comparison of the results obtained in a dynamical $\mathrm{n}$-SUSY scheme for the spectroscopic strengths in the ${ }^{196} \mathrm{Pt} \rightarrow$ ${ }^{195} \mathrm{Pt}$ reaction, using the boson-fermion expansion of the one nucleon transfer operator and truncating at different orders, shows the importance of going to higher order terms to describe experimental data, mainly in a scheme as restrictive as the dynamical n-SUSY.

We have performed the first comparison with experimental data of the results obtained using an IBFA boson number conserving image of the one nucleon transfer operator that includes the third term in the boson-fermion expansion and have shown that it produces good results for describing the experimental data of the ${ }^{196} \mathrm{Pt} \rightarrow{ }^{195} \mathrm{Pt}$ one neutron pickup reaction.

\section{ACKNOWLEDGMENTS}

Enlightening discussions with A. Frank and R. Bijker are acknowledged. This work has been partially supported by CONACYT, México, under Project No. 40027; DGI, Spain, under Project No. BFM2002-03315; and DFG, Germany, under Grant No. Jo391/2-1.
[1] F. Iachello, Phys. Rev. Lett. 44, 772 (1980).

[2] A. B. Balantekin, I. Bars, and F. Iachello, Nucl. Phys. A370, 284 (1981).

[3] P. Van Isacker, J. Jolie, K. Heyde, and A. Frank, Phys. Rev. Lett. 54, 653 (1985).

[4] A. B. Balantekin, Ph.D. thesis, Yale University, 1982.

[5] J. Jolie, Ph.D. thesis, University of Gent, 1986.
[6] J. Vervier, Riv. Nuovo Cimento 10, 1 (1987).

[7] A. Metz, J. Jolie, G. Graw, R. Hertenberger, J. Groger, C. Günther, N. Warr, and Y. Eisermann, Phys. Rev. Lett. 83, 1542 (1999).

[8] J. Groger, J. Jolie, R. Krücken, C. W. Beausang, M. Caprio, R. F. Casten, J. Cederkall, J. R. Cooper, F. Corminboeuf, L. Genilloud, G. Graw, C. Günther, M. de Huu, A. I. Levon, 
A. Metz, J. R. Novak, N. Warr, and T. Wendel, Phys. Rev. C 62 , 064304 (2000).

[9] A. Metz, Y. Eisermann, A. Gollwitzer, R. Hertenberger, B. D. Valnion, G. Graw, and J. Jolie, Phys. Rev. C 61, 064313 (2000).

[10] A. Metz, Y. Eisermann, A. Gollwitzer, R. Hertenberger, B. D. Valnion, G. Graw, and J. Jolie, Phys. Rev. C 67, 049901(E) (2003).

[11] F. Iachello and P. Van Isacker, The Interacting BosonFermion Model (Cambridge University Press, Cambridge, 1991).

[12] J. Barea, Ph.D. thesis, University of Seville, 2002.

[13] J. Barea, C. E. Alonso, and J. M. Arias, Phys. Rev. C 65, 034328 (2002).
[14] O. Scholten and A. E. L. Dieperink, in Interacting Bose-Fermi Systems in Nuclei, edited by F. Iachello (Plenum, New York, 1981), p. 343.

[15] O. Scholten, Prog. Part. Nucl. Phys. 14, 189 (1985).

[16] P. Van Isacker, A. Frank, and Hong-Zhou Sun, Ann. Phys. 157, 183 (1984).

[17] R. Bijker and O. Scholten, Phys. Rev. C 32, 591 (1985).

[18] Zhou Chumnei, Nucl. Data Sheets 71, 367 (1994).

[19] J. Jolie and P. Van Isacker, computer code IBFFMTR (unpublished).

[20] R. Bijker, computer codes ODDPAR and ODDSPEC, University of Groningen, 1983.

[21] J. Barea, computer code ODDPAR+ (modified ODDPAR), 1998 (unpublished). 